\title{
Study On The Mechanism of Color Formation In The Skin of Ophiopogon Japonicas Fruit Via Integrative Analysis of Metabolome And Transcriptome
}

\section{Sichen Gan}

Zhejiang Agriculture \& Forestry University

\section{Gang Zheng}

Zhejiang Agriculture \& Forestry University

\section{Fangfang Gui}

Zhejiang Agriculture \& Forestry University

Danying Lu

Zhejiang Agriculture \& Forestry University

Lijun Liang ( $\nabla$ llj@zafu.edu.cn )

Zhejiang Agriculture \& Forestry University

\section{Research Article}

Keywords: Ophiopogon japonicas, fruit color formation, anthocyanin, metabolome, transcriptome

Posted Date: August 26th, 2021

DOl: https://doi.org/10.21203/rs.3.rs-840343/v1

License: (9) This work is licensed under a Creative Commons Attribution 4.0 International License. Read Full License 


\section{Abstract}

In this study, the metabolome and transcriptome profiles of ophiopogon japonicas fruit during five stages were analyzed to understand the mechanisms for the formation of color in the skin. Results showed that twenty eight types of flavonoids existed in the skin of the fruit, of which twenty five were anthocyanins and three were flavonols. Among the twenty five kinds of anthocyanins, Delphinidin 3-0-rutinoside, Delphinidin 3-0-glucoside, Petunidin 3-0-rutinoside, Cyanidin 3-0-galactoside and Delphinidin 3-0galactoside were the major constitutes of the metabolites. Delphinidin 3-0-rutinoside and Delphinidin 3-0glucoside were discovered to be responsible for the blue color of fruit due to their high correlation with genes in the connection network, especially for Delphinidin 3-0-rutinoside, which was the highest in the amount and accumulated rapidly to $67.6 \%$ in the last three stages. Analyzing the results of co-expression network between genes and anthocyanins by means of weighted gene co-expression network analysis (WGCNA), the biosynthesis genes of anthocyanin formed in the late stage were found to be $F 3^{\prime} 5^{\prime} H, A N S$ and UFGT, and the regulatory gene was $M Y B$, which determined the color change of $O$. japonicas fruit. The identification of key anthocyanins and dominant genes for blue skin of $O$. japonicas fruit has laid a theoretical basis for regulating the fruit pigmentation in ground cover plants.

\section{Introduction}

As a kind of flavonoid ${ }^{1}$, anthocyanin consists of anthocyanidin backbone with sugar and acyl conjugates. More than 20 anthocyanidins have been discovered, but only six of them are prevalent in plants naming Cyanidin, Delphinidin, Pelargonidin, Malvidin, Peonidin and Petunidin. The color of anthocyanidin is dominated by the number of hydroxyl groups on the B-ring, and the more the hydroxyl groups, the bluer the color. In general, the blue color of plant tissue is related to the Delphinidin type anthocyanidin with two hydroxyl groups on the B-ring. Delphinidin type anthocyanins include Delphinidin and its derivatives Petunidin and Malvidin, of which the $3^{\prime}$ and $5^{\prime}$ positions of the B-ring are hydroxylated and/or methoxylated ${ }^{2}$. The accumulation of Delphinidin-type anthocyanins is the necessary but not the only requirement for developing blue/violet tissue. In some species, the interaction or participation of copigments and metal ions are also required ${ }^{3}$.

The biosynthesis pathway of anthocyanin is generally conserved in plants. The biosynthetic pathway for anthocyanin can be divided into two phases: phenylpropanoid and flavonoid, both are catalyzed by a series of enzymes. The early flavonoid biosynthesis genes (EBGs) are composed of phenylalanineammonia lyase (PAL), 4-coumaryl: CoA ligase (4CL), chalcone synthase (CHS), chalcone isomerase (CH) and flavanone 3-hydroxylase $(F 3 H)$, which encodes common precursor for various of flavonoids. The late anthocyanin biosynthesis genes (LBGs), such as flavonoid-3'-hydroxylase (F3'H), flavonoid-3', 5'hydroxylase $\left(F 3^{\prime} 5^{\prime} H\right)$, Dihydroflavonol 4-reductase (DFR), anthocyanidin synthase (ANS) and UDP-glucose: flavonoid 3-O-glucosyltransferase (UFGT), on the other hand, are closely relevant to the accumulation of anthocyanins ${ }^{4}$. Additionally, the accumulation of anthocyanins is highly depended on transcription factors (TFs) which regulates the expression of structural genes, and MYB-bHLH-WD40 complex (MBW) 
is the most common one in TFs. MYB as the most important protein of MBW normally determines the activation or inhibition characteristics of MBW. The $M Y B$ gene can independently regulate the structural genes among the anthocyanin biosynthesis pathway ${ }^{5-7}$ as well. Moreover, the expression of $M Y B$ gene in specific tissues can distinguish target gene promoters, otherwise, unusual expression of $M Y B$ gene often leads to abnormal accumulation of anthocyanins in plant ${ }^{8-10}$.

Integrating analysis of transcriptomics and metabolomics can give insight into transcriptional regulation and metabolic flux. To better know the mechanism of anthocyanin accumulation in fruits, the key genes are always screened and their correlation to the corresponding anthocyanin was disclosed ${ }^{11}$. For example, $\mathrm{Ni}$ et al. presented new regulatory patterns for phenotypic color among pears which controlled by jasmonate and ethylene ${ }^{12}$. Li et al. reported that the flavonoid homeostasis network, functioned as activation and feedback regulation of the flavonoids, determined the color of mulberry fruit, and the abnormal expression of $b H L H 3$ could lead to its color variation ${ }^{13}$. Based on weighted gene co-expression network analysis (WGCNA) of four fruit colors of pepper, Liu et al. deduced that the high expression of MYB113, WD68 and SLC40 resulted in significantly higher levels of flavonoids ${ }^{14}$. WGCNA as a systematic biological method, has the advantage in classifying the differentially expressed genes (DEGs) into several co-expression modules which accordingly helps identifying and obtaining hub genes from them ${ }^{15}$.

Ophiopogon japonicas belongs to Ophiopogoneae of Liliaceae family, and is widely used as both medicinal and ornamental plant. It is generally cultivated in the semi-shade of the forest margin or street lawn to cover the bare soil ${ }^{16,17}$. During the fruit season, 0 . japonicus fruit displays abundant and varying color of fruits from green to blue, which enriches the winter landscape, and accordingly, 0 . japonicus is also accepted as a superior fruit plant in garden plants. Tang studied the dynamic changes of total flavonoids and metal elements in 0 . japonicus fruits, and found that the amount of $\mathrm{Ca}^{2+}$ was high ${ }^{18}$. Cheng reported that the blue color of fruit extracts was greatly affected by $\mathrm{pH}$ value of the solution, which confirmed that anthocyanins were the main metabolites of 0 . japonicus fruits ${ }^{19}$. Anthocyanin dominant fruit were also found in other Ophiopogoneae species. Delphinin and Petunidin were obtained in blue fruit of 0 . jaburan, and the most effective co-pigment kaempferol was determined as well ${ }^{20-22}$. By now, four major anthocyanidins including two Delphinidins, one Petunidin and one Malividin have been identified in Liriope muscari black fruits ${ }^{23}$. However, few studies have been carried out on the gene regulation mechanism for the fruit color of Ophiopogoneae. It is of great significance to study the coloring mechanisms of 0 . japonicas fruit in order to elevating the ornamental performances of ground cover plants.

In this study, transcriptome and metabolome sequencing were performed on 0 . japonicas fruit sampling from five growth stages. 25 anthocyanins and 3 flavonols were obtained from specimens of the five stages. By analyzing the results of transcriptome and RT-qPCR, the expression profile of related genes for anthocyanin biosynthesis were identified. Based on dynamic changes of anthocyanins and genes, the crucial period for color changing was found. The key genes and anthocyanins which responsible for the 
blue skin of 0 . japonicas fruits were finally screened out through analyzing the correlation between genes and metabolites. This study not only revealed the mechanisms for anthocyanin accumulation and regulation in the skin of 0 . japonicas fruit from the aspects of transcriptomics and metabolomics, but also provided important insights in the molecular network of ornamental fruits, which founded molecular basis for the color cultivation of ornamental fruit.

\section{Materials And Methods}

\section{Plant materials and sampling}

O. japonica is widely planted in China, and accordingly, it is not restricted by any relevant legislation or guidelines. Fruits of $\mathrm{O}$. japonicas were collected from a germplasm garden situated at Zhejiang A\&F University, Hangzhou, China ( $29^{\circ} 56^{\prime}$ to $30^{\circ} 23^{\prime}$, E $118^{\circ} 51^{\prime}$ to $119^{\circ} 52^{\prime}$ ), and the collection of plant material was allowed by college for experimental research. The fruits were collected evenly from the scape of fifteen to twenty plants located at the same area. Five growth stages for the collection were set based on the color and size of fruits. The first stage (S1) was arranged on September of 2020, followed by the second (S2) on October, the third (S3) on November, the fourth (S4) on December and the fifth (S5) on January, 2021. All the freshly picked fruits were frozen immediately in liquid nitrogen after sampling, and stored at $-80^{\circ} \mathrm{C}$ in Zhejiang Provincial Key Laboratory of Germplasm Innovation and Utilization for Garden Plants, Key Laboratory of National Forestry and Grassland Administration on Germplasm Innovation and Utilization for Southern Garden Plants, School of Landscape Architecture, Zhejiang Agriculture \& Forestry University, Hangzhou 311300, China.

\section{Determination on the Pigment content}

$200 \mathrm{mg}$ fruit from the assigned growth stage was firstly ground into powder in $200 \mu \mathrm{l}$ absolute ethyl alcohol (EA), then sealed and preserved in dark place. When the fruits turned colorless, the chlorophyll was considered to be dissolved in supernatant liquid. After filtrating the crushed fruits, the filter was collected and ready to be determined. The absorbance of extraction was measured at $470 \mathrm{~nm}, 649 \mathrm{~nm}$, and $665 \mathrm{~nm}$, respectively. The content of chlorophyll was computed according to the method described by $\mathrm{Hu}^{43}$. The total anthocyanin was extracted at $4^{\circ} \mathrm{C}$ in dark by a mixed solution which was formulated as $\mathrm{EA}: \mathrm{H}_{2} \mathrm{O}: \mathrm{HCl}=80: 20: 0.3$ (calculated as volume, $\mathrm{mL}$ ) ${ }^{32}$. The extracts were measured the absorbance $530 \mathrm{~nm}$ and $600 \mathrm{~nm}$. The relative content (amount) of total anthocyanin (TAC) was computed according to Liu's method ${ }^{44}$.

\section{Sample of metabonomics preparation and disposition}

The freeze-dried sample was crushed with zirconia beads using a mixer mill (MM 400, Retsch) at $30 \mathrm{~Hz}$ for $1.5 \mathrm{~min} .50 \mathrm{mg}$ powder was vortexed in $0.5 \mathrm{ml}$ methanol/water/hydrochloric acid (799:200:1,V/V/V) for $10 \mathrm{~min}$ and ultrasonically extracted for $10 \mathrm{~min}$. The extracts were then centrifuged at 12, $000 \mathrm{rpm}$ 
under $4^{\circ} \mathrm{C}$ for 3 min to allow phase separation. The extraction was repeated twice and the total supernatants were filtrated and collected (PTFE, $0.22 \mu \mathrm{m}$; Anpel) for LC-MS/MS analysis.

\section{UPLC Conditions}

The extracts were analyzed using UPLC-ESI-MS/MS system (UPLC, Shim-pack UFLC SHIMADZU CBM30A system, www.shimadzu.com.cn/; MS, Applied Biosystems 6500 Triple Quadrupole, www.appliedbiosystems.com.cn/), and the conditions were as follows. The WatersACQUITY BEHC18 (1.7 $\mu \mathrm{m}, 2.1 \mathrm{~mm} * 100 \mathrm{~mm})$ column and a mobile phase composed of water $(0.1 \%$ formic acid) and methanol ( $0.1 \%$ formic acid) was used for separation under the following gradient conditions: $95: 5 \mathrm{~V} / \mathrm{V}$ initially, $50: 50 \mathrm{~V} / \mathrm{V}$ at $6 \mathrm{~min}, 5: 95 \mathrm{~V} / \mathrm{V}$ at $12 \mathrm{~min}$, holding for $2 \mathrm{~min}, 95: 5 \mathrm{~V} / \mathrm{V}$ at $14 \mathrm{~min}$, holding for $2 \mathrm{~min}$. The flow rate was $0.35 \mathrm{~mL} / \mathrm{min}$, with the column temperature at $40^{\circ} \mathrm{C}$ and injection volume of $2 \mu \mathrm{L}$. The effluent was alternatively connected to an ESI-triple quadrupole-linear ion trap (QTRAP)-MS.

\section{ESI-Q TRAP-MS/MS}

Linear ion trap and triple quadrupole (QQQ) scans were acquired by a triple quadrupole-linear ion trap mass spectrometer (Q TRAP). API 6500 Q TRAP UPLC/MS/MS System, equipped with an ESI Turbo lonSpray interface, was operated in positive and negative ion mode and controlled by Analyst 1.6.3 software (AB Sciex). The ESI source operation parameters were as follows: ion source with turbo spray, source temperature at $550^{\circ} \mathrm{C}$; ion spray voltage (IS) at $5500 \mathrm{~V}$ (positive ion mode), curtain gas (CUR) at $35 \mathrm{psi}$, and the highly collision active dissociation (CAD). Each ion pair was scanned according to the optimized declustering potential (DP) and collision energy (CE). The metabolites eluted in each period were quantified using Multiple Reaction Monitoring (MRM).

\section{Total RNA extraction and quality analysis}

Total RNA was extracted by RNA extraction kit (TIANGEN RNAprep FFPE Kit) according to the corresponding specifications. The integrity of RNA was detected by Agilent 2100 Bioanalyzer (Agilent Technologies Inc., CA, USA) and agarose gel electrophoresis, while the purity and concentration of the RNA were analyzed by a NanoDrop ${ }^{\text {TM }}$ One/OneC system (Thermo Fisher Scientific, MA, USA).

\section{Iso-Seq library construction and Data Analysis}

Total RNA of the five stages was maximized to acquire the full-length transcriptome. Iso-Seq library was prepared according to the Isoform Sequencing protocol (Iso-Seq) and using the Clontech SMARTer PCR cDNA Synthesis Kit protocol as described by Pacific Biosciences(www.pacb.com, PN 100-092-800-03). After PacBio system sequencing, $33.75 \mathrm{~Gb}$ subreads were obtained. SMRTlink 7.0 software was used to process these sequence data as follows. The circular consensus sequences (CCS) were generated from subreads of BAM files, and the resulted CCS contained full length non-chimeric (FLNC) sequences. The final isoforms were gained from high-quality consensus FLNC reads after removing the redundant FLNC through Quiver and CD-HIT-v4.6.754, respectively. 


\section{Construction of Illumina RNA-seq library and estimation on expression level}

RNA sequences of fruits from five growth stages, with three replicates for each stage were concluded, which means a total number of fifteen libraries were established. Each sequencing library was prepared with $1 \mu \mathrm{g}$ RNA and was generated from NEBNext ${ }^{\circledR}$ UltraTM RNA Library Prep Kit of Illumina ${ }^{\circledR}$ (NEB, USA). The quantitation and quality of library was detected by Qubit2.0 Fluorometer and Agilent 2100 bioanalyzer, respectively. When passing the quality inspection, the library preparations were sequenced on an Illumina Novaseq platform and 150 bp paired-end reads were generated.

The expression level of each gene was determined by the fragments-per-kilobase of transcript-per-millionmapped reads (FPKM), which was calculated by featureCounts v1.5.0-p3. Differential expression analysis between two stages was performed using the DESeq2 R package (1.16.1). The obtained P-values were then adjusted according to the Benjamini and Hochberg's approach for controlling the false discovery rate. Genes with an adjusted P-value $<0.05$ detected with DESeq2 were designated as differentially expressed genes (DEGs) (fold-change $\geq 2$, false discovery rate $<0.01$ ).

\section{qRT-PCR analysis}

The extraction of RNA was carried out according to the method mentioned above and PrimerScript ${ }^{\mathrm{TM}} \mathrm{RT}$ Master Mix cDNA (TaKaRa, Japan) was used for cDNA synthesis. The primer pairs for each isoform/unigene were designed using primer 5 . The obtained cDNA samples were analyzed by qRT-PCR in a $20 \mu$ reacting solution formulated with TB Green ${ }^{\text {TM }}$ Premix Ex Taq ${ }^{\text {TM }}$ (TaKaRa, Japan), primer pairs and double distilled water. The qRT-PCR analysis was performed on a Light-Cycler 480 Il real-time PCR detection system (Roche, USA).

\section{Statistical analysis}

SIMCA 14 was applied to conduct the Principal Component Analysis (PCA) and Variable Importance in Projection (VIP). One-way ANOVA was prformed by means of Duncan's multiple range test of the Statistical Product and Service Solutions program (version 19) (SPSS Inc.,Chicago, IL), and the graphs were plotted using Graphpad prism 8.0.

\section{Results}

\section{Relationship between the color development and the content variation of chlorophyll and anthocyanin}

The growth of 0 . japonicas fruits was divided into five stages according to the color of skin and size of fruit. Figure. 1a-c presented the growth of fruit, dimension of long axis (length) and the hundred-grain weight (HGW). During S1 and S2, green was the dominant color of the fruit and it became darker from S1 to S2. S3 was the transition period for color development and when entering the fourth stage, blue turned to be the prevailing color. From S4 to S5, the blue turned deeper. The average length of fruit on S1was as 
low as $5.83 \pm 0.20 \mathrm{~mm}$, but increased continuously by 1.11 -fold, 1.48 -fold and 1.09 -fold from S1 to S2, S2 to S3 and S3 to S4, respectively, then leveled off from S4 to S5. The variation of HGW had similar

tendency as the length. It grew from $3.38 \pm 0.23 \mathrm{~g}$ on the first stage to $11.07 \pm 0.60 \mathrm{~g}$ on the fifth stage, the weight gain of which increased significantly from stage 1 to stage $4(P<0.05)$ and slowed down from stage 4 to stage 5 .

The content of chlorophyll and anthocyanin accumulated in the skin of 0 . japonicas fruits was displayed in Fig. 1 d. The amount of chlorophyll climbed rapidly from $14.38 \pm 1.62 \mu \mathrm{g} / \mathrm{g} \mathrm{FW}$ to $25.50 \pm 2.84 \mu \mathrm{g} / \mathrm{g} \mathrm{FW}$ during the first two stages and declined quickly to the lowest from S2 to S4. On the contrary, the content of total anthocyanin was near zero during the first two stages, but soared in the following three stages, reaching the highest in S4, followed by a slight drop on S5. Interestingly, the relative content of chlorophyll was equivalent to that of anthocyanin around stage three. When correlating the proportion of chlorophyll and anthocyanin to the color of the fruit, it is understandable that the fruit developed from green dominant color to blue prevailing color, with a transition period appeared around stage three. Hereby, the phenotypic changes of $O$. japonicas fruits skin were highly consistent with the content of anthocyanin and chlorophyll.

\section{The Metabolites profiles of 0 . japonicas Fruits skin}

The fruits of 0 . japonicus were collected from the five growth stages and the composition of the skin was analyzed by means of metabolome. 28 types of flavonoids were obtained, of which 25 was classified as anthocyanin compounds and 3 was flavonols. Among the 25 anthocyanin compounds, 6 was the main and most common anthocyanins, which proved that $O$. japonicas fruits contained complete biosynthesis pathway for the anthocyanin. Further study on the accumulation of the 6 types of anthocyanin compounds and flavonol compounds during every growth stage was conducted and the results were presented in Fig. 1-e. It is evident that Delphinidin derivatives were the major metabolites, which accounted near $80 \%$ in the last three stages. Principal component analysis (PCA) was adopted to investigate the metabolites of the five growth stages and the results were shown in Fig. 2. Samples from S1 and S2 were obviously clustered, which confirmed that there was no significant change in the type and amount of metabolites during the first two stages. But the following three stages could be defined clearly through PC1 explaining $66.4 \%$ and PC2 explaining 12.6\%. Based on the analyses above, S3 was considered to be the key period for color variation of fruits.

\section{Differentially Accumulated Metabolites (DAMs) Analysis of O. japonicas Fruit skin}

To better understand the major metabolites affecting the coloration of 0 . japonicas Fruit, differentially accumulated metabolites (DAMs) analysis was applied under the condition of VIP $\geq 1, p<0.05$, Log2FC $\geq 1$ or Log2FC $\leq-1$. A total number of 21 flavonoids were found which included 19 anthocyanins and 2 flavonols. The heat maps (Fig. 3. a) showed that most anthocyanins increased sharply in the amount during S3 and leveled off during S5, of which Delphinidin 3-0-rutinoside, Delphinidin 3-0-glucoside, Petunidin 3-0-rutinoside, Cyanidin 3-0-galactoside and Delphinidin 3-0-galactoside varied dramatically and became the major metabolites at the ripening stage, accordingly, they were considered to be the key 
metabolites for the transition of fruit color from green to blue. In addition, among the five anthocyanins, Delphinidin 3-0-rutinoside was the most abundant one, which accounted for $69.2 \%$ of the DAMs in S4, 95.57 times higher than that at $\mathrm{S} 1$.

The expression profiles of flavonols, on the other hand, were similar to anthocyanins in the first four stages, but decreased significantly at $\mathrm{S} 5$, which could be the key metabolites to distinguish the last two stages. The content of Quercetin 3-0-glucoside and Dihydromyricetin declined rapidly at S5, 7.05-fold and 2.39-fold lower, respectively, in comparison with S4.

\section{Gene expression patterns of flavonoid biosynthesis}

Based on KEGG pathway, 293 genes regulating anthocyanin and flavonol biosynthesis were identified, and 181 of them was differentially expressed (Table 1). As shown in Fig. 3. b, a part of EBGs became low in expression during the fruit ripening stages, which mainly involved in eight $P A L s$, two $C 4 H s$, two $C H S s$, five $\mathrm{CH} / \mathrm{s}$ and one $\mathrm{F} 3 \mathrm{H}$. In comparison, the LBGs including DFRs, ANSs, UFGTs and OMTs, shared the same expression profile with anthocyanins except one DFR and five OMTs. Owing to the high expression, LBGs were considered to be positively correlated with the accumulation of anthocyanin. In addition to the biosynthesis genes, the transcription factors (TFs) also affected the accumulation of anthocyanin. Therefore, a total of 817 TFs regulatory genes assigned to 67 gene families were identified, of which 351 belonged to DEGs. The major TFs of MYBs and bHLHs, which control the biosynthesis of anthocyanins, were focused on and their expression levels were analyzed in detail. The expression level of 7 MYBs was found to be consistent with the accumulation of anthocyanins, and three of them including maidong_transcript_11129, maidong_transcript_7157 and maidong_transcript_6578 were highly expressed in S3. Nevertheless, $b H L H$ gene (maidong_transcript_6829), the only differentially expressed, after reaching the highest expression level in S2 decayed gradually in the subsequent periods (Fig. 3. C). 
Table 1

Candidate unigenes involved in anthocyanin biosynthesis, flavonol biosynthesis in 0 . japonicas fruit skin.

\begin{tabular}{|c|c|c|c|c|c|}
\hline & Genes & Description & DEGs & & ALL \\
\hline \multirow[t]{6}{*}{ ( } & $P A L$ & phenylalanine ammonia-lyase & 29 & & 45 \\
\hline & $\mathrm{C} 4 \mathrm{H}$ & trans-cinnamate 4-monooxygenase & 19 & & 28 \\
\hline & $4 C L$ & 4-coumarate-CoA ligase & 5 & & 5 \\
\hline & $\mathrm{CHS}$ & chalcone synthase & 22 & & 28 \\
\hline & $\mathrm{CHI}$ & chalcone isomerase & 19 & & 30 \\
\hline & $\mathrm{F} 3 \mathrm{H}$ & naringenin 3-dioxygenase & 6 & & 6 \\
\hline \multirow[t]{5}{*}{ ( } & $F 3^{\prime} 5^{\prime} H$ & flavonoid 3',5'-hydroxylase & 6 & & 7 \\
\hline & $D F R$ & dihydroflavonol 4-reductase & 5 & & 5 \\
\hline & LDOX/ANS & leucoanthocyanidin dioxygenase & 9 & & 10 \\
\hline & UFGT & anthocyanidin 3-0-glucosyltransferase & 8 & & 15 \\
\hline & OMT & caffeoyl-CoA 0-methyltransferase & 25 & & 32 \\
\hline \multirow[t]{2}{*}{ प } & $F L S$ & flavonol synthase & 3 & & 3 \\
\hline & FG3 & flavonol-3-0-glucoside & 5 & & 6 \\
\hline \multirow[t]{2}{*}{ 口 } & $M Y B$ & transcription factor MYB & & 19 & 53 \\
\hline & $b H L H$ & transcription factor bHLH & & 1 & 20 \\
\hline
\end{tabular}

Obviously, the FLSs and FG3s genes, which are vital for flavonol biosynthesis expressed strongly at S3 and S4, and correspondingly the content of Quercetin 3-0-glucoside and Dihydromyricetin was high in the fruit. The expression level at S5 was significantly lower than in S4, which were consistent with the variation of flavonols.

\section{Identifying anthocyanin-related DEGs by Co-expression network analysis}

4004 DEGs (with mean FPKM > 45) were selected in this study to perform WGCNA. According to the standard WGCNA networks, the soft power was set as 13, min Module Size as 30 and the merge Cut Height as 0.15 . The DEGs were clustered into 11 tree branches, and each one represented a module displaying different color (Fig. 4., Fig. 5.). The highly related DEGs in the same module were co-expressed according to the standard WGCNA, but the color of Cyanidin 3-0-glucoside corresponding to the modules 
were different from those of the other DAMs, which suggested that the gene regulatory network of Cyanidin 3-0-glucoside was independent. Further analysis revealed that six modules were highly positively correlated to DAMs $(P<0.05)$, while three modules were highly negatively correlated to DAMs $(P<0.05$, Table 2$)$. Based on the analyses above, the centrality weights of modules nodes were obtained by means of CentiScaPe (http://apps.cytoscape.org/apps/centiscape\#cy-app-releases-tab), that was, by calculating the geometric means of degree centrality, closeness centrality and betweenness centrality with the edge weights between 0.2 and 0.8 , the Hub genes were then screened from the highest centrality weight. Among the positive modules, hub genes that were predicted to encode the synthesis enzymes of anthocyanins were more abundant in purple module (Fig. 6.), including five ANSs

(maidong_transcript_18463, maidong_transcript_14175, maidong_transcript_12015, maidong_transcript_20037 and maidong_transcript_22160), F3'5'H (maidong_transcript_2898) and UFGT (maidong_transcript_9469). The maidong_transcript_6056 (unknown protein) identified as hub gene in purple module was highly correlated to the anthocyanin synthesis genes including $C H S$, three ANSS, UFGT and $F 3^{\prime} 5^{\prime} H$, which suggested that maidong_transcript_6056 gene played an important role in the synthesis of anthocyanin. Except for purple module, four hub genes including $\mathrm{C} 4 \mathrm{H}$ (maidong_transcript_3366), two OMTs (maidong_transcript_18253 and maidong_transcript_9185)and GSTImaidong_transcript_22603凸sourced from pink module, greenyellow module and midnightblue

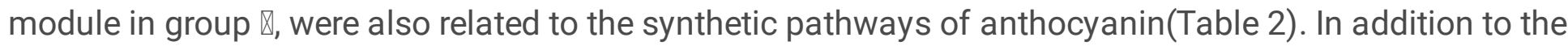
genes described above, two isoflavone 2'-hydroxylase $\left(I 2^{\prime} H\right)$ genes (maidong_transcript_3111 and maidong_transcript_3164) ascribed to midnightblue module were found to be homologous to soybean $g m / 2^{\prime} H^{24}$, but their expression increased continuously during the five stages, indicating that the synthesis of isoflavone was slightly different from that of anthocyanin.

Table 2

Statistics of module-traits with high correlations.

\begin{tabular}{|c|c|c|c|}
\hline Group & Module & Response & DEGs \\
\hline प & greenyellow $\llbracket$ midnightblue $\llbracket p i n k \rrbracket p u r p l e \llbracket b l a c k \llbracket g r e e n$ & Positively respond to DAMs & 1488 \\
\hline Q & turquoise $\llbracket$ cyan $\square$ red & Negatively respond to DAMs & 2025 \\
\hline
\end{tabular}

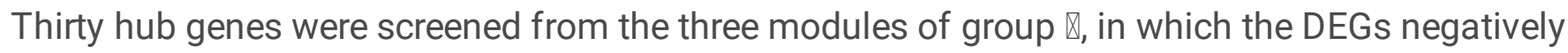
responded to DAMs (Table 2), and 9 of hub genes were annotated in KEGG. Among the nine genes, LHCB1 (light-harvesting Complex II chlorophyll A/B binding protein 1), which belonged to the red module and encoded light-harvesting Complex protein, participated in the photosynthesis. The expression of $\angle H C B 1$ that directly affected the content of chlorophyll ${ }^{25}$ declined continuously during the five stages, and the expression level dropped 35.01 times at S3 in comparison with S1. Maidong_transcript_13873, as the hub gene of cyan module, was annotated as caltractin protein, one of the Ca-binding protein, which displayed a continuous drop in gene expression from S2 to S5. However, hub genes of turquoise module which negatively respond to DAMs were not annotated in KEGG.

Relationship between gene expression and the amount of anthocyanin in O. japonicas fruit skin 
In order to investigate the gene regulatory network of anthocyanins biosynthesis in 0 . japonicas fruits, five anthocyanins and 181 DEGs (Table 1) profiles were selected and the Pearson's correlation coefficients were calculated. Correlations with a coefficient of $\mathrm{R}^{2}>0.8$ were adopted to visualize a network, which was displayed in Fig. 7. There were 5 anthocyanins and 89 DEGs in the diagram, including 75 structural genes and 14 regulatory genes. The highest centralities assigned to the module's nodes were obtained according to the method described in co-network. DEGs and DAMs included in the six nodes were two UFGTS (maidong_transcript_3906 and maidong_transcript_13974), two regulatory genes MYBs (maidong_transcript_7157 and maidong_transcript_6578) and two anthocyanins (Delphinidin 3-0rutinoside and Delphinidin 3-0-glucoside), respectively. Among the six highly correlated DEGs and DAMs, the correlation between two MYBs and UFGTS (maidong_transcript_3906) reached 0.8841 and 0.9090 , respectively. In addition, the expression levels of the two MYBs were closely related to the accumulation of Delphinidin-types anthocyanin. When analyzing their profile in the fruits, the variation of these candidate nodes was basically similar, and their expression level or content were significantly increased in S3, which suggested that these DEGs and DAMs were responsible for color formation of 0 . japonicas fruit.

\section{Validation via qRT-PCR}

Based on the results of WGCNA and the correlation between DAMs and DEGs, qRT-PCR was conducted on 11 hub genes screened from purple module (Fig. 6.) and co-network (Fig. 7.). Taking RNA-binding protein Musashi (MSI) and ubiquitin-fold modifier conjugating enzyme 1 (UFC1) as the reference genes, the 11 hub genes for the 0 . japonicas fruits at five stages were verified and presented in Fig. 8. a-k. To validate the accuracy of RNA-seq analysis, the coefficient of Pearson's correlation was computed. Except for one MYB gene (maidong_transcript_6578), the expression patterns of RNA-seq and RT-qPCR were highly consistent, especially for $A N S s$, and the $\mathrm{R}^{2}$ ranged from 0.7543 to 0.9824 with $\mathrm{p}<0.05$.

The expression level of 11 hub genes was low or scarce during the first two periods. When entering S3, the expression level climbed gradually, and the accumulation of anthocyanin promoted the transition of 0 . japonicas fruit from green to blue. This gene expression reached the peak at $\mathrm{S} 4$, and the content of anthocyanin also attained the maximum. Subsequently in S5, the expression level of genes dropped rapidly except for one $M Y B$ (maidong_transcript_6578), and the accumulation of anthocyanin ceased. Based on the analyses above, MYB, ANS, UFGT and $F 3^{\prime} 5^{\prime} H$ were considered to be the key types of genes for anthocyanin synthesis.

\section{Discussion}

The color of plant organs is generally controlled by the type and amount of anthocyanins. Delphinin was reported to be the most common anthocyanin in blue organs of plant ${ }^{26}$. Studies on the mature fruit of 0 . japonicas also revealed that Delphinin pigments were dominant, followed by Petunidins, which agreed with the blue color of its fruit and confirmed that large proportion of Delphinin was the requirement for blue organ ${ }^{27}$. When the content of Petunidin and Malvidin increases, the carboxylation and/or 
methoxylation reactions happened on the 3 ' and 5 ' positions of the B-ring of Delphinin reduced the reactivity of hydroxyl groups ${ }^{28}$, consequently, the color shifts red slightly ${ }^{29}$. Alternatively, when the content of Cyanidin is equivalent to that of Delphinin, the petal or fruit often shows purple phenotypes 30,31 . Glycosylation of Delphinidins on the 3' position hydroxyl group occurred commonly in plants, but was crucial to develop blue fruits of 0 . japonicas. This phenomenon also existed in most blue/violet organs, such as the plant Gentiana scabra and blueberry ${ }^{32-34}$. Glycosylation of Delphinidins occuring on the 3' position of the B-ring, including Delphinidin 3-0-rutinoside and Delphinidin 3-0-glucoside were consistent with phenotypic variation of $O$. japonicas fruits and the expression profile of DEGs. In comparison, Delphinidin 3-0-rutinoside was dominant and responsible for the blue color of 0 . japonicas fruits, which was also reported in blue flower of Tulipa gesneriana cv. Murasakizuisho ${ }^{3}$.

The synthesis of Anthocyanin is regulated by a variety of structural genes, and it is difficult to identify key gene only through analyzing the expression profile of DEGs. Therefore, WGCNA, as a mean of dividing coexpression DEGs into many modules, was introduced in this experiment. Hub genes were identified as the highest correlation in each module and could represent the expression features of the module ${ }^{35}$. In positive modules that correlated with anthocyanin $(P<0.05)$, the most LBGs that identified as hub genes enriched in purple module, including $F 3^{\prime} 5^{\prime} H$, three $A N S s$, and UFGT. RT-qPCR validation confirmed that dynamic changing between relative quantitation and FKPM of these genes were similar. Based on the analyses above, $F 3^{\prime} 5^{\prime} H, A N S$ and UFGT were considered as the key genes for the accumulation of Delphinidin, which was proved in other blue/violet plants. $F 3^{\prime} 5^{\prime} H$ was located at the first node of anthocyanin biosynthesis pathway, and when it competed with $F 3^{\prime} H$ for dihydrokaempferol, a metabolic intermediate, Delphinidin and Cyanidin will be generated. The $F 3^{\prime} 5^{\prime} H$ gene is so important that plant will fail to produce blue/purple flowers without it ${ }^{36}$. The expression of $F 3^{\prime} 5^{\prime} H$ was enhanced from S3 laid foundation for the accumulation of Delphinidin in the skin of $O$. japonicas fruits. However, only high expression of $F 3^{\prime} 5^{\prime} H$ was not enough. ANS and UFGT as LBGs were also required for the synthesis of anthocyanin, and the expression level of which determined the color of organs. A total of 181 DEGs related to anthocyanin biosynthesis were identified by KEGG pathway, the LBGs of which expressed increasingly during S3 except one DFR and five OMTs. Combined with the analysis of WGCNA, ANS, UFGT and $F 3^{\prime} 5^{\prime} H$ were confirmed as the hub genes, the expression levels of which were highly consistent with the variation of Delphinin content. It was confirmed that $L B G s$ regulated the accumulation of anthocyanin in 0 . japonicas fruits, which was similar with previous studies. For example, Chen reported that the color of eggplant petal was controlled by a single dominant gene called Flower Anthocyanidin Synthase(FAS), which was encoded into the ANS protein. A single base-pair deletion of FAS at the 438th position will lead to transcription halt and the failure of anthocyanin accumulation, as a result, the plant produced mutant with white-flower ${ }^{37}$. Zhang confirmed that $A N S$ was a key gene in Hosta plantaginea purple petals ${ }^{38}$. Under the catalyzation of $A N S$, anthocyanidins will produce exposed hydroxyl groups, which could be modified via glycosylation and the formation of intramolecular bonds made them stable under acidic conditions. UFGT, as a modifier gene, also significantly affects the accumulation of blue/violet anthocyanins. It is reported that the mechanism for exogenous calcium signal to induce the 
accumulation of the anthocyanin in Vitis vinifera was the interaction between calmodulin and UFGT ${ }^{39}$. Noda succeeded in cultivating blue chrysanthemum by introducing UGFT, whereas non-glycogenated transgenic chrysanthemums showed purple ${ }^{40}$. These results suggested that the high expression of $F 3^{\prime} 5^{\prime} H$, ANS and UFGT genes was essential for the accumulation of Delphinin.

In addition to LBGs, TFs also regulate the accumulation of Anthocyanin, of which MYB is the most common and critical one. In this research, correlation analyses disclosed that MYB gene located at the center of the regulatory network (Fig. 7.) and was co-expressed with the key gene UFGT (maidong_transcript_3906). Moreover, the expression profile of MYB gene was closely related to the content of Delphinidin 3-0-rutinoside and Delphinidin 3-0-glucoside. These results were consistent with the previous studies. Masumi reported that MYB19LONG in Lilium brownii was the essential gene involving in producing anthocyanin pigmentation, which was stimulated by different vascular bundle signals to develop anthocyanin pigmentation related brushmarks or raised spots ${ }^{41}$. Experiments of SSMYB1 conducted by overexpression in Arabidopsis thaliana and VIGS silence in Sapium sebiferum leaves confirmed that the accumulation of anthocyanins in $S$. sebiferum leaves was depended on $S S M Y B 7^{42}$. However, the variation of $M Y B$ gene expression always causes abnormal anthocyanin accumulation in fruit (e.g., Citrus grandis ${ }^{8}$, 'Fuji' apple (Ralls Janet $\times$ Red Delicious) ${ }^{9}$ and Elaeis guineensis ${ }^{10}$ ). Therefore, it was speculated that MYB protein activated the expression of LBGs, leading to the accumulation of Delphinidin in 0 . japonicas fruits.

\section{Conclusions}

In summary, the blue skin of $O$. japonicas fruits was originated from Delphinidin, and of which, Delphinidin 3-0-glucoside and Delphinidin 3-0-rutinoside were the key metabolic substances for the color transformation. In addition, the transcription factor $M Y B$ gene and downstream genes LBGs including $F 3^{\prime} 5^{\prime} H$, ANS and UFGT were the key genes for anthocyanin biosynthesis. These results will not only serve the purpose for elevating fruit pigmentation of $O$. japonicas and the other proximal species, but also provide important ways to the identification of related genes and proteins involved in blue fruit development.

\section{Declarations}

\section{Author Contributions}

L.L. and S.G. designed the study; S.G., G.Z., F.G., and D.L. carried out the experiments and contributed to the interpretation of the results; S.G. wrote the manuscript; L.L. supervised and critically revised the manuscript.

\section{Acknowledgements}

Not applicable. 


\section{Competing interests}

Conflicts of Interest: The authors declare that they have no conflict of interest

\section{References}

1. Tanaka, Y., Sasaki, N. \& Ohmiya, A. Biosynthesis of plant pigments: Anthocyanins, betalains and carotenoids. Plant Journal, 54, 733-749 https://doi.org/10.1111/j.1365-313X.2008.03447.x (2008).

2. Noda, N. Recent advances in the research and development of blue flowers. Breeding Science, 68, 79-87 https://doi.org/10.1270/jsbbs.17132 (2018).

3. Shoji et al. Perianth Bottom-Specific Blue Color Development in Tulip cv. Murasakizuisho Requires Ferric lons. Plant and Cell Physiology, 48, 243-251 https://doi.org/10.1093/pcp/pcl060 (2007).

4. Xia, D. et al. How rice organs are colored: the genetic basis of anthocyanin biosynthesis in rice. The Crop Journal, 21, https://doi.org/10.1016/j.cj.2021.03.013 (2021).

5. WenjiaXu, C. D. \& LoïcLepiniec Transcriptional control of flavonoid biosynthesis by MYB-bHLH-WDR complexes. CellPress, 20, 176-185 https://doi.org/10.1016/j.tplants.2014.12.001 (2015).

6. Kanzaki, S. et al. The R2R3-MYB transcription factor MiMYB1 regulates light dependent red coloration of 'Irwin' mango fruit skin. Scientia Horticulturae, 272, 109567 https://doi.org/10.1016/j.scienta.2020.109567 (2020).

7. Ma, D. \& Constabel, C. P. MYB Repressors as Regulators of Phenylpropanoid Metabolism in Plants. Trends in Plant Science, 24, 275-289 https://doi.org/10.1016/j.tplants.2018.12.003 (2019).

8. Ding et al. Subfunctionalization of the Ruby2-Ruby 1 gene cluster during the domestication of citrus. Nature Plants, 4, 930-943 https://doi.org/10.1038/s41477-018-0287-6 (2018).

9. Hye et al. Differential gene expression and epigenetic analyses between striped and blushed skinned sports of 'Fuji' apple. Scientia horticulturae, 261, 108944-108944 https://doi.org/10.1016/j.scienta.2019.108944 (2020).

10. Singh, R. et al. The oil palm VIRESCENS gene controls fruit colour and encodes a R2R3-MYB. Nature Communications, 5, 4106 https://doi.org/10.1038/ncomms5106 (2014).

11. Xue, Q. et al. Transcriptomics and targeted metabolomics profilings for elucidation of pigmentation in Lonicera japonica flowers at different developmental stages. Industrial Crops and Products, 145, 111981 https://doi.org/10.1016/j.indcrop.2019.111981 (2020).

12. Ni, J. et al. Ethylene mediates the branching of the jasmonate-induced flavonoid biosynthesis pathway by suppressing anthocyanin biosynthesis in red Chinese pear fruits. Plant Biotechnology Journal, 18, 1223-1240 https://doi.org/10.1111/pbi.13287 (2020).

13. Li, H. et al. Abnormal expression of bHLH3 disrupts a flavonoid homeostasis network, causing differences in pigment composition among mulberry fruits. Horticulture Research, 7, 83 https://doi.org/10.1038/s41438-020-0302-8 (2020). 
14. Liu, Y. et al. Integrative analysis of metabolome and transcriptome reveals the mechanism of color formation in pepper fruit (Capsicum annuum L.). Food Chemistry, 306, 125629 https://doi.org/10.1016/j.foodchem.2019.125629 (2020).

15. Du, J. et al. Identification of regulatory networks and hub genes controlling soybean seed set and size using RNA sequencing analysis. Journal of Experimental Botany, 68, 1955-1972 https://doi.org/10.1093/jxb/erw460 (2017).

16. Li, J. W. \& Li, X. P. Study on the geographical distribution and Flora of the Ophiopogon in China. Hunan Forestry Science \& Technology, 25, 26-30 doi:CNKI:SUN:HLKJ.0.1998-01-006 (1998).

17. Ru, W. Escription on Wild Flower Resources of Species Liliaceae (Ophiopogon, Liriope) in the Western of Henan. Horticulture \& Seed, 37, 22-24 https://doi.org/10.16530/j.cnki.cn21-1574/s.2017.07.009 (2017).

18. Tang, C. Study on medicinal components in fruits of Ophiopogon japonicus. Journal of Taiyuan Teachers College (Natural Science Edition), 4, 69-72 https://doi.org/10.3969/j.issn.16722027.2005.01.020 (2005).

19. Chen, C. et al. Study on Stability of Blue Pigment from Ophiopogon japonicus Fruit. Food Science, 29, 168-171 https://doi.org/10.3321/j.issn:1002-6630.2008.12.034 (2008).

20. Ishikura, N. \& Hayashida, S. Kaempferol Glycosides in the Seed-Coat of Ophiopogon jaburan (Kunth) Lodd. Agricultural and Biological Chemistry, 43, 1923-1926 https://doi.org/10.1080/00021369.1979.10863726 (1979).

21. Ishikura, N. \& Hayashida, S. A new petunidin tetraglycoside and tulipanin from Ophiopogon seeds. The Botanical Magazine Tokyo, 93, 89-94 https://doi.org/10.1007/BF02489115 (1980).

22. Ishikura, N. \& Yoshitama, K. Anthocyanin-Flavonol Co-pigmentation in Blue Seed Coats of Ophiopogon jaburan. Journal of Plant Physiology, 115, 171-175 https://doi.org/10.1016/s01761617(84)80064-4 (1984).

23. Choung, M. G. et al. Antimelanogenic effect and whitening of anthocyanin rich fraction from seeds of Liriope platyphylla. Korean Journal of Medicinal Crop Science, 21, 361-371 https://doi.org/10.7783/KJMCS.2013.21.5.361 (2013).

24. Chenglin et al. Soybean transcription factor ORFeome associated with drought resistance: a valuable resource to accelerate research on abiotic stress resistance. BMC genomics, 16, 1-9 https://doi.org/10.1186/s12864-015-1743-6 (2015).

25. Liu, H. et al. Comparative analysis of the photosynthetic physiology and transcriptome of a highyielding wheat variety and its parents. The Crop Journal, 8, 1037-1048 https://doi.org/10.1016/j.cj.2020.01.004 (2020).

26. He, J. J., Liu, Y. X., Pan, Q. H., Cui, X. Y. \& Duan, C. Q. Different Anthocyanin Profiles of the Skin and the Pulp of Yan73 (Muscat Hamburg $\times$ Alicante Bouschet) Grape Berries. Molecules, 15, 1141-1153 https://doi.org/10.3390/molecules15031141 (2010).

27. Escher, G. B., Wen, M., Zhang, L., Rosso, N. D. \& Granato, D. Phenolic composition by UHPLC-Q-TOFMS/MS and stability of anthocyanins from Clitoria ternatea L. (butterfly pea) blue petals. Food 
Chemistry, 331, 127341 https://doi.org/10.1016/j.foodchem.2020.127341 (2020).

28. Kim, B. G. et al. Characterization of an 0-methyltransferase from soybean. Plant Physiology and Biochemistry, 44, 236-241 https://doi.org/10.1016/j.plaphy.2006.05.003 (2006).

29. Tatsuzawa, F., Tanikawa, N. \& Nakayama, M. Red-purple flower color and delphinidin-type pigments in the flowers of Pueraria lobata (Leguminosae). Phytochemistry, 137, 52-56 https://doi.org/10.1016/j.phytochem.2017.02.004 (2017).

30. Lou, Q. et al. Transcriptome sequencing and metabolite analysis reveals the role of delphinidin metabolism in flower colour in grape hyacinth. Journal of Experimental Botany, 65, 3157-3164 https://doi.org/10.1093/jxb/eru168 (2014).

31. Tatsuzawa, F. Flower colors and flavonoids in the cultivars of Lobelia erinus L. Dyes and Pigments, 180, 108500 https://doi.org/10.1016/j.dyepig.2020.108500 (2020). Campanulaceae

32. Hutabarat, R. P. et al. Identification of anthocyanins and optimization of their extraction from rabbiteye blueberry fruits in Nanjing. Journal of Food Quality. 2019, 1-10, doi:10.1155/2019/6806790 (2019).

33. Stein-Chisholm, R., Beaulieu, J., Grimm, C. \& Lloyd, S. LC-MS/MS and UPLC-UV Evaluation of Anthocyanins and Anthocyanidins during Rabbiteye Blueberry Juice Processing. Beverages, 3, 56 https://doi.org/10.3390/beverages3040056 (2017).

34. Tasaki, K., Higuchi, A., Watanabe, A., Sasaki, N. \& Nishihara, M. Effects of knocking out three anthocyanin modification genes on the blue pigmentation of gentian flowers. Scientific Reports, $\mathbf{9}$, 15831 https://doi.org/10.1038/s41598-019-51808-3 (2019).

35. Ding, T. et al. Identification of gene co-expression networks and key genes regulating flavonoid accumulation in apple (Malus $\times$ domestica) fruit skin. Plant Science, 304, 110747 https://doi.org/10.1016/j.plantsci.2020.110747 (2020).

36. DAl, S. \& Dai, H. Molecular Breeding for Flower Colors Modification on Ornamental Plants Based on the Mechanism of Anthocyanins Biosynthesis and Coloration. Scientia Agricultura Sinica, 49, 529542 https://doi.org/10.3864/j.issn.0578-1752.2016.03.011 (2016).

37. Chen, M. et al. Fine Mapping Identifies SmFAS Encoding an Anthocyanidin Synthase as a Putative Candidate Gene for Flower Purple Color in Solanum melongena L. International Journal of Molecular Sciences, 19, 789 https://doi.org/10.3390/ijms19030789 (2018).

38. Zhang, J. et al. Transcriptome-Wide Analysis Reveals Key DEGs in Flower Color Regulation of Hosta plantaginea (Lam.) Aschers. Genes (Basel), 11, 31 https://doi.org/10.3390/genes11010031 (2019).

39. Jun Yu, M. Z., Wang, M., Xu, Y., Chen, W. \& Yang, G. Transcriptome analysis of calcium-induced accumulation of anthocyanins in grape skin. Scientia Horticulturae, 260, 108871 https://doi.org/10.1016/j.scienta.2019.108871 (2020).

40. Naonobu Noda et al. Generation of blue chrysanthemums by anthocyanin B-ring hydroxylation and glucosylation and its coloration mechanism. ScienceAdvances, 3, e1602785 https://doi.org/10.1126/sciadv.1602785 (2017). 
41. Yamagishi, M. MYB19LONG is involved in brushmark pattern development in Asiatic hybrid lily (Lilium spp.) flowers. Scientia Horticulturae, 272, 109570

https://doi.org/10.1016/j.scienta.2020.109570 (2020).

42. Chen, $X$. et al. The R2R3-MYB transcription factor SsMYB1 positively regulates anthocyanin biosynthesis and determines leaf color in Chinese tallow (Sapium sebiferum Roxb.). Industrial Crops and Products, 164, 113335 https://doi.org/10.1016/j.indcrop.2021.113335 (2021).

43. Hu, B. et al. Evaluation of the optimum concentration of chlorophyll extract for determination of chlorophyll content by spectrophotometry. Pratacultural Science, 35, 1965-1974 https://doi.org/10.11829/j.issn.1001-0629.2017-0418 (2018).

44. Liu, T. et al. Transcriptome analyses reveal key genes involved in skin color changes of 'Xinlimei' radish taproot. Plant Physiology and Biochemistry, 139, 528-539 https://doi.org/10.1016/j.plaphy.2019.04.006 (2019).

\section{Figures}


(a)

$\underline{5 \mathrm{~mm}}$

(b)

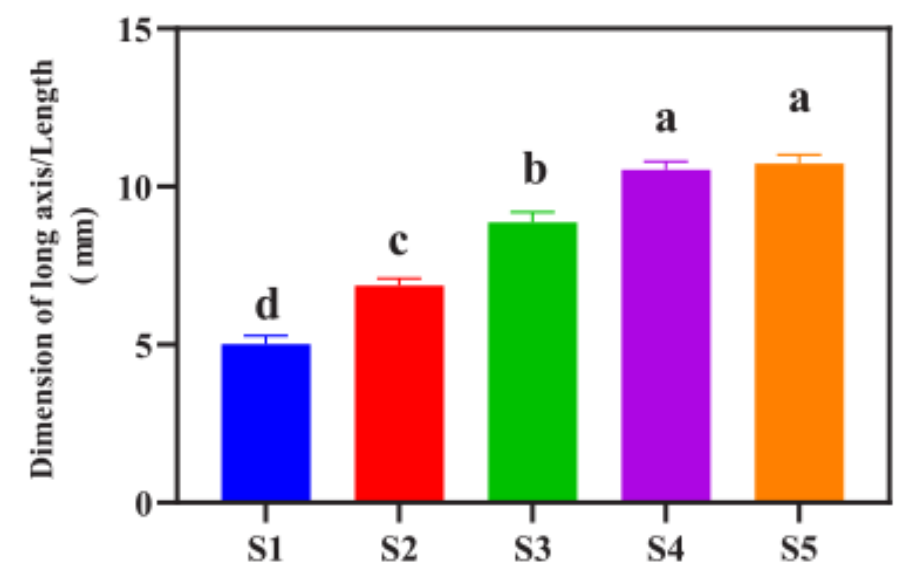

(d)

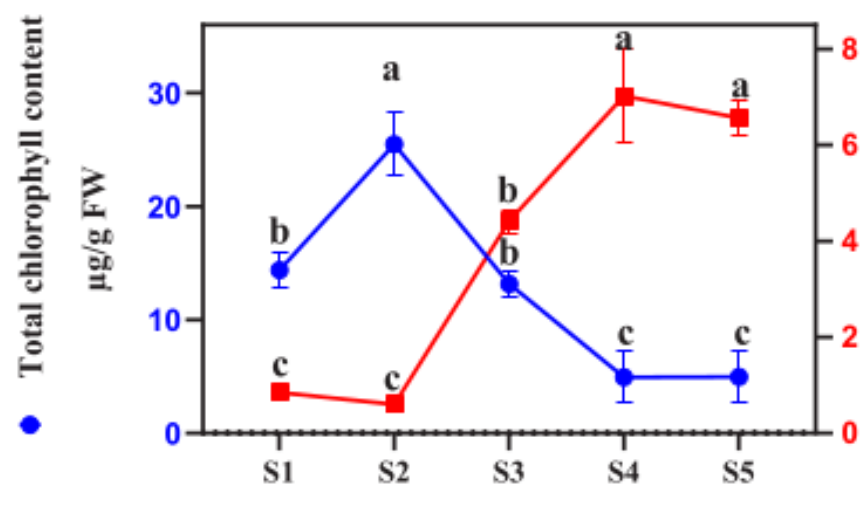

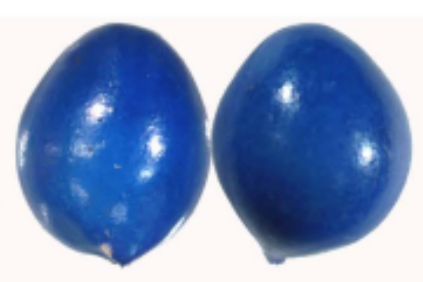

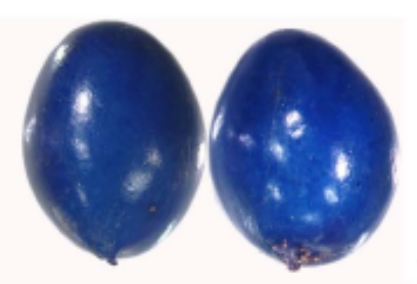

(c)

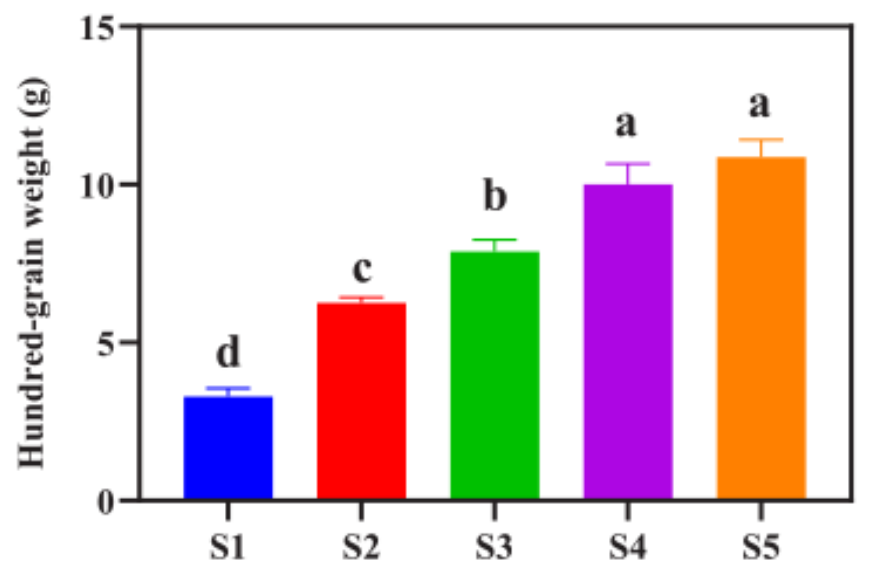

(e)

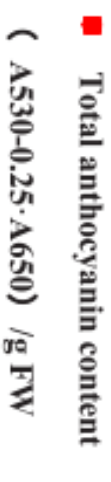

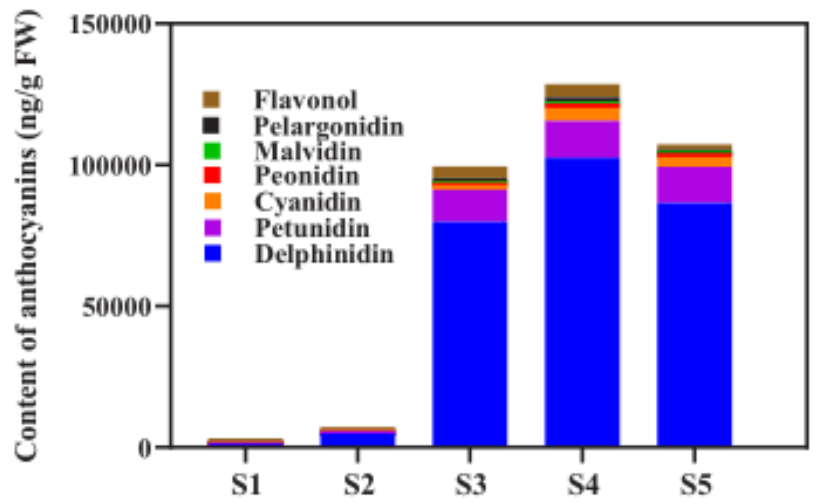

Figure 1

Phenotypic changing and metabolite changing of 0 . japonicas fruits during five developmental stages.

(a) the changing of color, (b) the lenth, (c) the hundred-grain weight, (d) the Chlorophyll and total anthocyanin during five stages. (e) Various of anthocyanin-types and flavonol content profile during five stages. The different letters represent significant differences, $P<0.05$. 


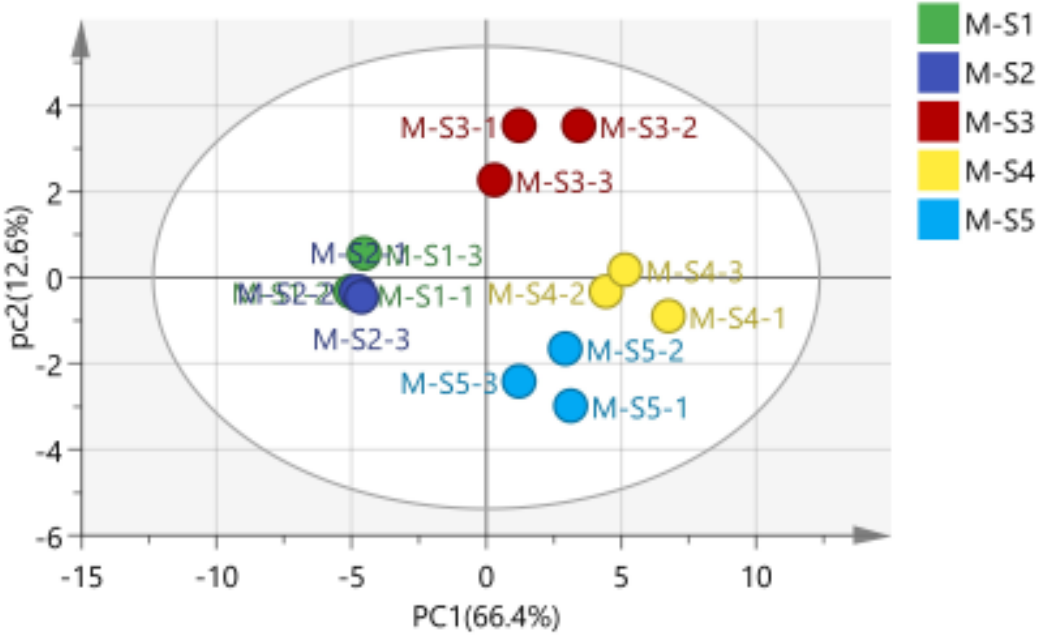

Figure 2

PCA score plot of each 0. japonicas fruits sample.

(a)

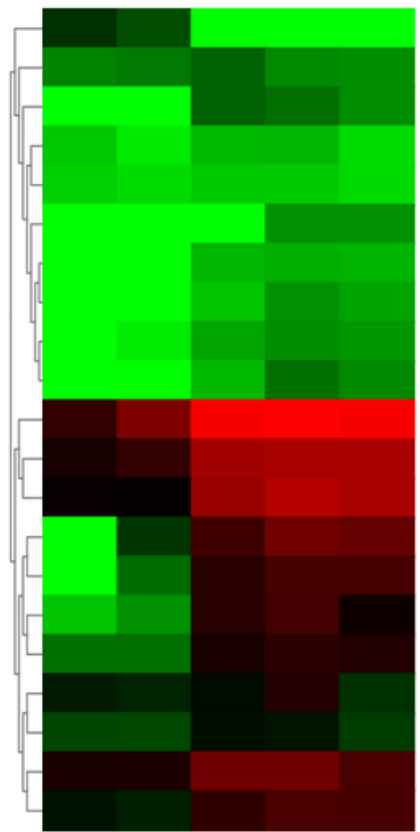

$\begin{array}{lllll}\text { S1 } & \text { S2 } & \text { S3 } & \text { S4 } & \text { S5 }\end{array}$

(C)

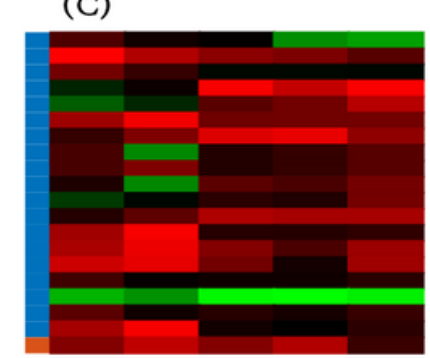

$\begin{array}{lllll}\text { S1 } & \text { S2 } & \text { S3 } & \text { S4 } & \text { S5 }\end{array}$
Cyanidin 3-O-glucoside

Cyanidin 3-O-(6-O-malonyl-beta-D-glucoside) Pelargonidin 3-O-(6-O-malonyl-beta-D-glucoside) Pelargonidin 3,5-O-diglucoside Malvidin 3-O-arabinoside Peonidin 3-O-galactoside Petunidin 3-O-arabinoside Malvidin 3-O-(6-O-malonyl-beta-D-glucoside) Delphinidin 3-O-arabinoside Peonidin 3,5-O-diglucoside Delphinidin 3-O-rutinoside Petunidin 3-O-rutinoside Delphinidin 3-O-glucoside Cyanidin 3-O-galactoside Delphinidin 3-O-galactoside Pelargonidin 3-O-rutinoside Cyanidin 3-O-arabinoside Quercetin 3-O-glucoside Pelargonidin 3-O-glucoside Dihydromyricetin Peonidin 3-O-rutinoside

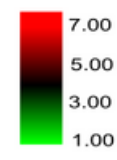

\section{Group \\ MYB
bHLH}

(b)
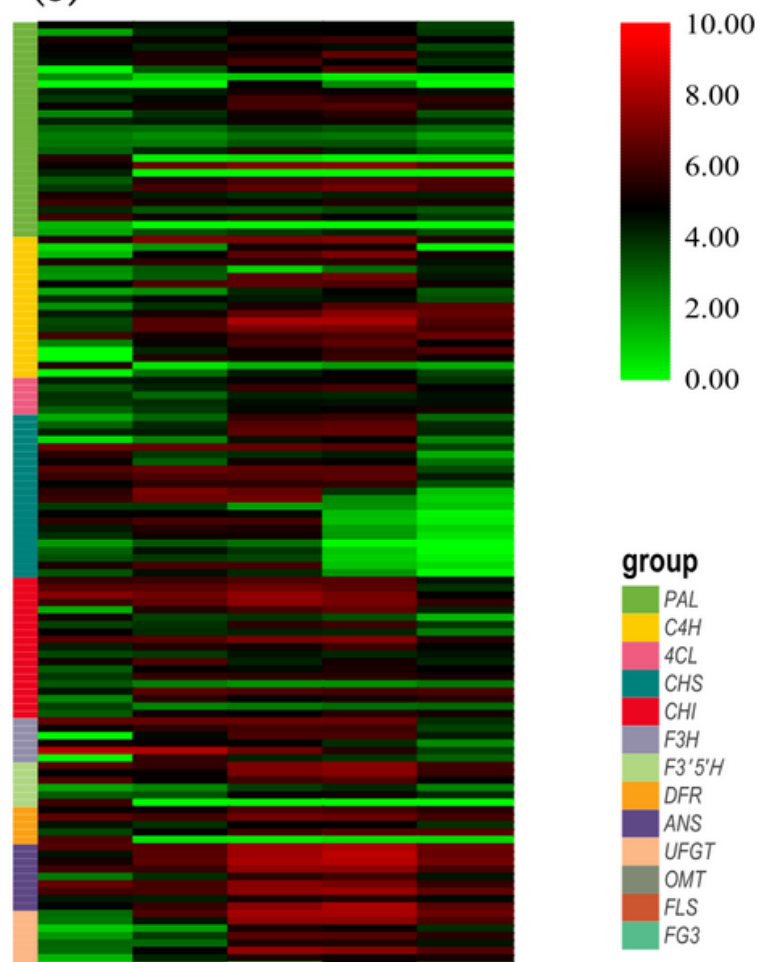

Figure 3 
The dynamic profile of DEGs and DAMs. (a) The heat map of DAMs, (b) EBGs and LBGs and (c) MYB and bHLH genes in 0 . japonicas fruits five stages.

\section{Cluster Dendrogram}
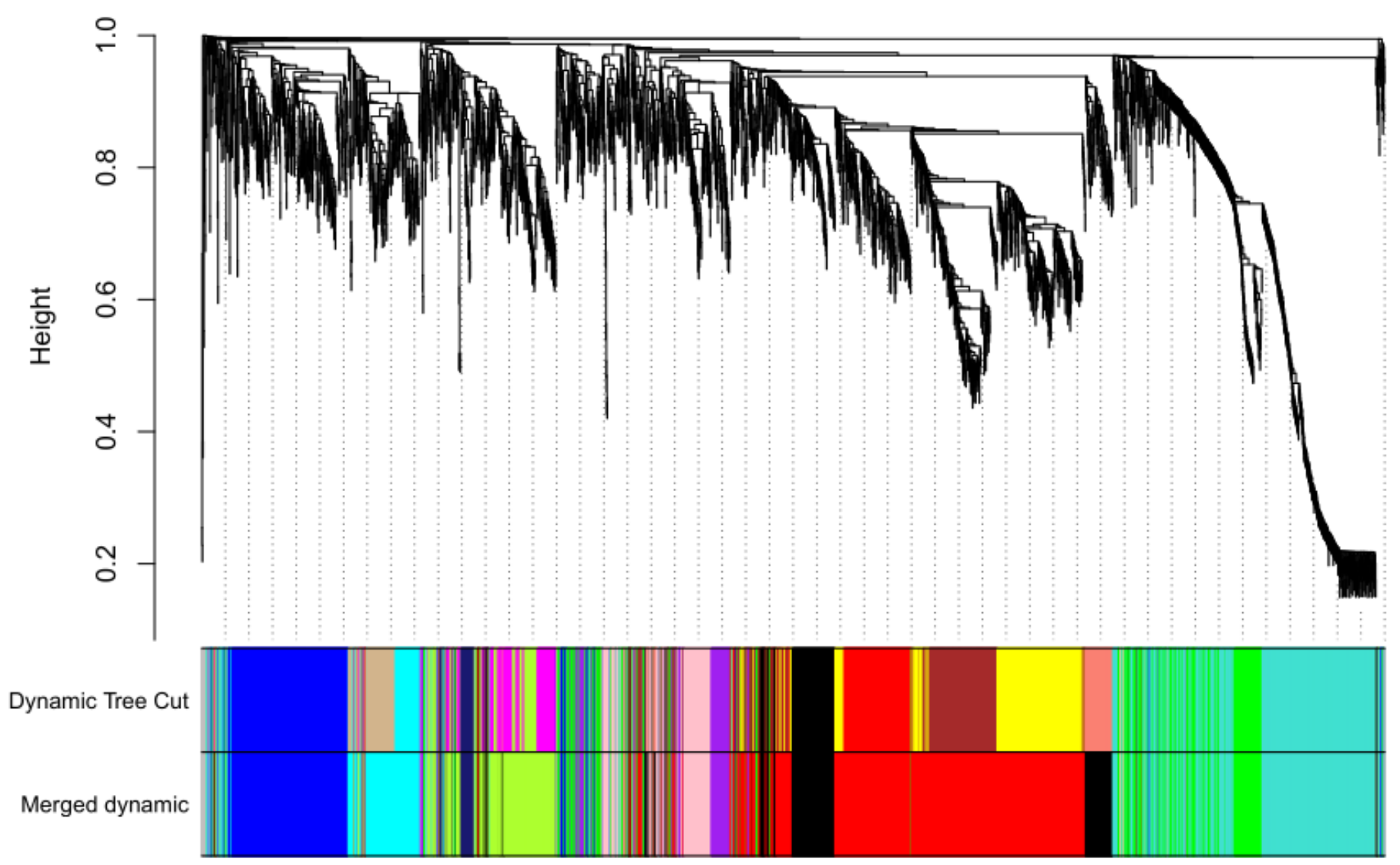

Figure 4

Dendrogram of 4004 DEGs obtained via hierarchical clustering of topologic al overlapping dissimilarity. 


\section{Module-trait relationships}

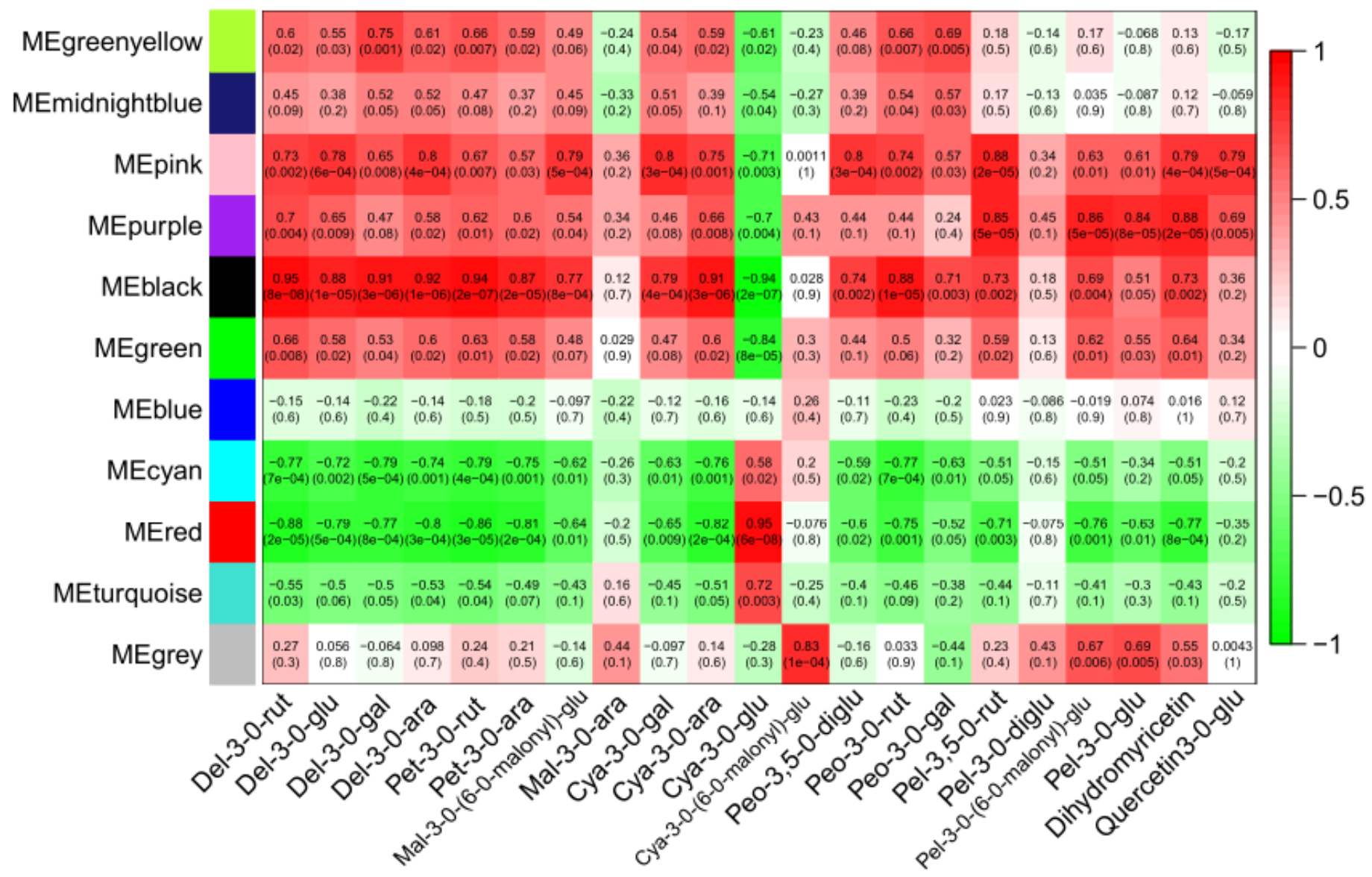

Figure 5

Relationships between module eigengenes (ME, rows) and traits as DAMs (columns). 


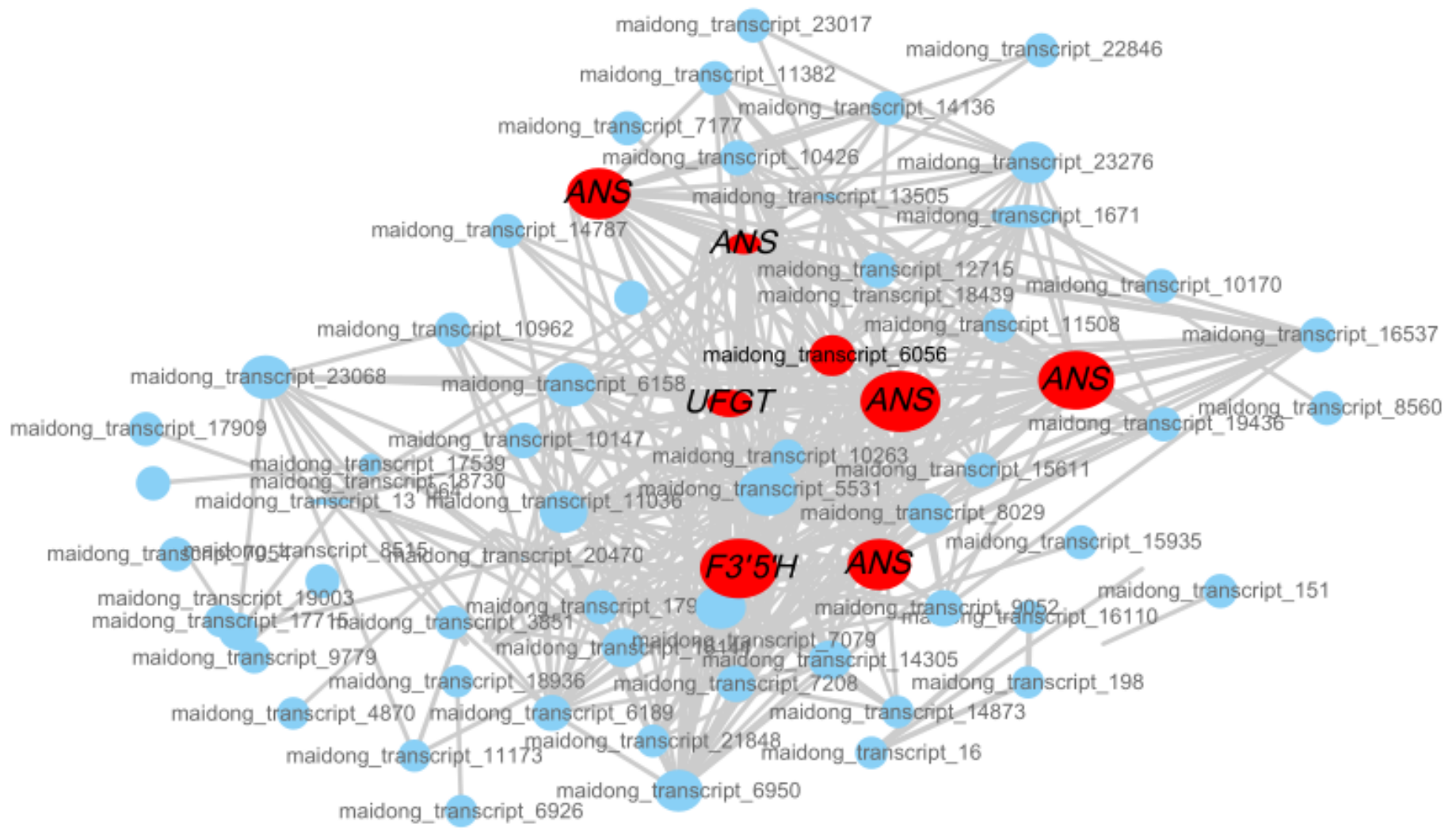

Figure 6

Correlation networks in the purple module. 


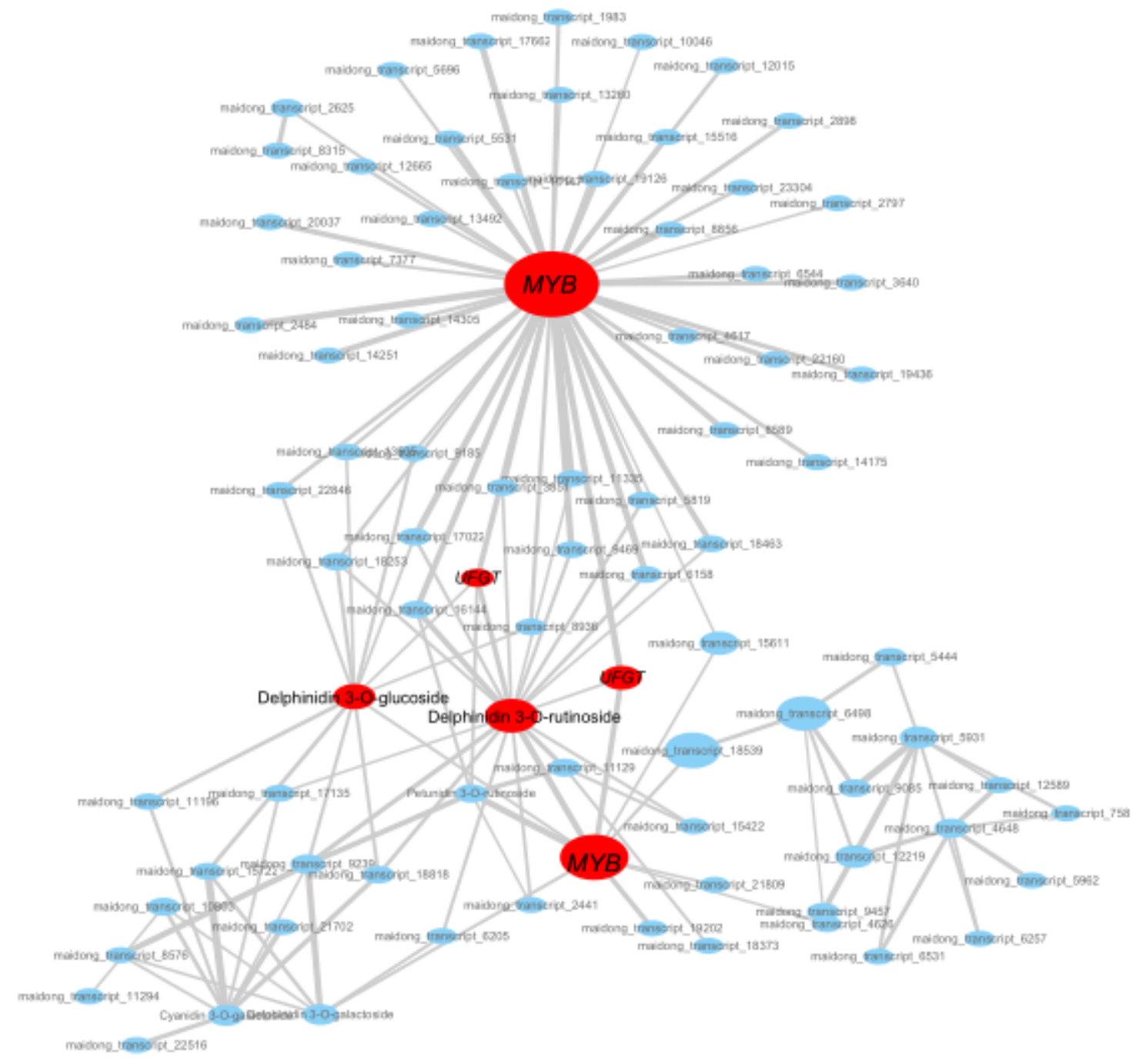

Figure 7

The correlation networks of 5 major anthocyanins and 89 DEGs, including 75 structural genes and 14 regulatory genes. 

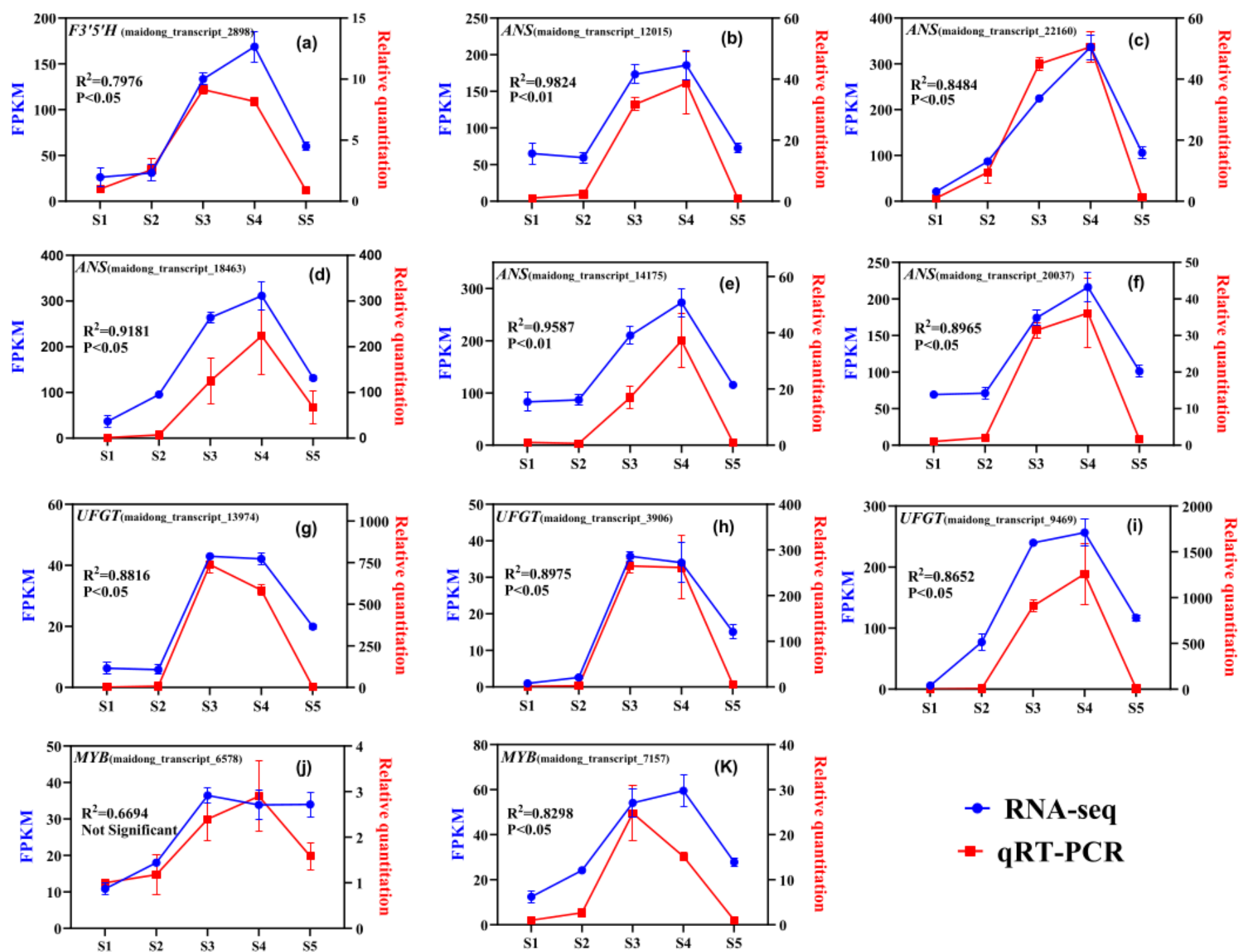

\section{RNA-seq}

qRT-PCR

\section{Figure 8}

RT-qPCR results of eleven selected hub genes. 